\title{
Tracing the origins of genotype VIIh Newcastle disease in Southern Africa
}

Running title: Exotic Newcastle disease outbreaks in Southern Africa

Celia Abolnik, ${ }^{1}$ Chrisborn Mubamba, ${ }^{2,3}$ Daniel B.R. Wandrag, ${ }^{1}$ Roger Horner, ${ }^{4}$ Bruce Gummow, ${ }^{2}$

George Dautu, $^{3}$ Shahn P.R. Bisschop ${ }^{5}$

${ }^{1}$ Department of Production Animal Studies, Faculty of Veterinary Science, University of Pretoria, Pretoria, South Africa

${ }^{2}$ Discipline of Veterinary Sciences, College of Public Health Medical and Veterinary Sciences James

Cook University, Queensland, Australia. QLD 4811

${ }^{3}$ Ministry of Fisheries and Livestock, Department of Veterinary Services, Zambia

${ }^{4}$ Midlands Poultry Health Centre, Howick, KwaZulu Natal, South Africa.

${ }^{5}$ Avimune (Pty) Ltd. Pretoria, South Africa. 


\section{SUMMARY}

It is widely accepted that Newcastle disease is endemic in most African countries, but little attention has been afforded to establishing the sources and frequency of the introductions of exotic strains. Newcastle disease outbreaks have a high cost in Africa, particularly on rural livelihoods. Genotype VIIh emerged in Southeast Asia and has since caused serious outbreaks in poultry in Malaysia, Indonesia, southern China, Vietnam and Cambodia. Genotype VIlh reached the African continent in 2011, with the first outbreaks reported in Mozambique. Here, we used a combination of phylogenetic evidence, molecular dating and epidemiological reports to trace the origins and spread of sub-genotype VIIh Newcastle disease in Southern Africa. We determined that the infection spread northwards through Mozambique, and then into the poultry of the north-eastern provinces of Zimbabwe. From Mozambique, it also reached neighbouring Malawi and Zambia. In Zimbabwe, the disease spread southward towards South Africa and Botswana, causing outbreaks in backyard chickens in early to mid-2013. In August 2013, the disease entered South Africa's large commercial industry, and the entire country was infected within a year, likely through fomites and the movements of cull chickens. Illegal poultry trading or infected waste from ships and not wild migratory birds was the likely source of the introduction to Mozambique in 2011.

Key words: Africa, Newcastle disease virus, epidemiology, molecular clock 


\section{INTRODUCTION}

Newcastle disease (ND) is a highly contagious and frequently lethal disease of birds caused by an Avulavirus in the Paramyxoviridae family. The enveloped virus contains a negative sense, singlestranded RNA genome and primarily replicates in the respiratory and gastrointestinal tract. Thus, Newcastle disease virus (NDV) is able to spread between infected and susceptible birds through aerosolised oral/nasal secretions and oral/faecal contact. Clinical signs and the severity of the disease depend on a range of factors including host species, age, immune status and viral characteristics, although gastrointestinal, respiratory and neurological symptoms are typical (Alexander, 2000).

All NDV strains belong to a single serotype but multiple genotypes and sub-genotypes have emerged over the course of several decades, driven in some part by intensive farming with increasingly high densities of poultry and the use of vaccines. Two classes of virus (I and II) have been defined, but all virulent strains implicated in ND panzootics have emerged from Class II. Class II is further divided into eighteen genotypes (I to XVIII), some containing sub-genotypes, based on genetic distances between viral fusion glycoprotein gene sequences (Diel et al., 2012). Viruses from Genotype VII have been responsible for the most recent ND panzootic. It originated in Southeast Asia, with the earliest known outbreaks beginning around 1985. Sub-genotypes VIla to VIIk are presently described (Diel et al., 2012; Molini et al 2017).

Genotype VIIh emerged in Southeast Asia and has since been identified as the cause of outbreaks in poultry in Malaysia since 2004 (Tan et al., 2010; Choi et al., 2014), Indonesia since 2007 (Adi et al., 2010; Xiao et al., 2012), southern China since 2011 (Xie et al 2012), Vietnam since 2011 (Choi et al, 2014) and Cambodia since 2011 (Choi et al., 2013). The intra-cerebral pathogenicity index (ICPI) in day-old chicks was measured at 1.81 to 1.86 out of a maximum score of 2.0 , confirming the high virulence of numerous genotype VIIh strains (Choi et al., 2013). Genotype VIIh reached the African continent in 2011. An outbreak in vaccinated broiler chickens in December 2011 was detected in 
Maputo province, Mozambique. Between 2012 and 2016 the infection spread from the Maputo province to the Sofala and Zambesia provinces, where outbreaks in vaccinated birds were associated with mortality rates of up to $71 \%$. The outbreak strains were phylogenetically linked to viruses from China, Southeast Asia and a single isolate from South Africa in 2013 (Mapaco et al, 2016). We report the detection and molecular characterization of genotype VIIh in South Africa, Malawi, Zambia and Zimbabwe. Apart from South Africa, few strains are available for comparison, therefore a combination of epidemiological reports, phylogenetic data and inference of recent common ancestors (RCAs) using time-stamped viral sequences was used to trace the origins and spread of exotic genotype VIIh in the southern African region. 
Table 1: Viral genetic sequences used for phylogenetic reconstruction and estimation of recent common ancestors

\begin{tabular}{|c|c|c|c|}
\hline Isolate & Region & Sampling date & $\begin{array}{l}\text { Accession } \\
\text { number }\end{array}$ \\
\hline NDV/Bali-1/07 (genotype VIIa) & Indonesia & January 2007 & AB605247 \\
\hline Chicken/Makassar/003/09 & Indonesia & 2009 & HQ697256 \\
\hline Chicken/Bali/020/10 & Indonesia & 2010 & HQ697261 \\
\hline Chicken/Sukorejo/019/10 & Indonesia & 2010 & HQ697255 \\
\hline UPM-IBS/002/2011 & Malaysia & 2011 & KF026013 \\
\hline IBS005/11 & Malaysia & 2011 & KR074405 \\
\hline Egret/China/Guangxi/2011 & southern China & May 2011 & JX193074 \\
\hline NDV/chicken/Mozambique/1205/2011 & Mozambique & December 2011 & KX231366 \\
\hline Chicken/Guizhou/1031/2012 & Southwest China & April 2012 & KU175230 \\
\hline Chicken/Guizhou/1032/2012 & Southwest China & April 2012 & KT760568 \\
\hline Chicken/Guizhou/1176/2012 & Southwest China & 2012 & KU175232 \\
\hline NDV/chicken/Mozambique/466/2012 & Mozambique & June 2012 & KU523524 \\
\hline NDV/chicken/Mozambique/491/2012 & Mozambique & June 2012 & KU523526 \\
\hline KH-cmb08-2/12 & Cambodia & June 2012 & JX870619 \\
\hline KH-cmb06-01/12 & Cambodia & June 2012 & JX870617 \\
\hline NDV/chicken/Mozambique/658/2012 & Mozambique & July 2012 & KU523528 \\
\hline Goose/Yunnan/1200/2013 & China & 2013 & KU175233 \\
\hline NDV/chicken/Mozambique/584/2013 & Mozambique & June 2013 & KU523529 \\
\hline Chicken/South Africa/230665/2013 ${ }^{\mathrm{a}}$ & South Africa & August 2013 & MF622035 \\
\hline Turkey/South Africa/N2057/2013 ${ }^{\text {a }}$ & South Africa & October 2013 & KR815908 \\
\hline
\end{tabular}




\begin{tabular}{|c|c|c|c|}
\hline Chicken/Zimbabwe/235280/2013 ${ }^{\mathrm{a}}$ & Zimbabwe & October 2013 & MF622036 \\
\hline Chicken/South Africa/RBNW-1/2013 & South Africa & November 2013 & MF622045 \\
\hline Chicken/South Africa/RBNW-2/2013 & South Africa & November 2013 & MF622046 \\
\hline Chicken/South Africa/RBNW-3/2013 ${ }^{\text {a }}$ & South Africa & November 2013 & MF622047 \\
\hline Chicken/South Africa/Inchanga/2013 & South Africa & November 2013 & MF622040 \\
\hline Chicken/South Africa/239391/2013 ${ }^{a}$ & South Africa & November 2013 & MF622037 \\
\hline NDV/chicken/Mozambique/475/2014 & Mozambique & July 2014 & KX231367 \\
\hline NDV/chicken/Mozambique/622/2014 & Mozambique & August 2014 & KU523533 \\
\hline Chicken/South Africa/H14973/2014 ${ }^{\mathrm{a}}$ & South Africa & September 2014 & MF622039 \\
\hline Chicken/South Africa/32995/2015 a & South Africa & July 2015 & MF622034 \\
\hline Chicken/South Africa/N2117/2015 & South Africa & August 2015 & MF622043 \\
\hline Chicken/South Africa/N2683/2015 & South Africa & November 2015 & MF622044 \\
\hline Chicken/Zambia/Katete/2015 ${ }^{\text {a }}$ & Zambia & November 2015 & MF622041 \\
\hline Chicken/Zambia/Mbeweka/2015 ${ }^{\text {a }}$ & Zambia & November 2015 & MF622042 \\
\hline Chicken/Zambia/Chadiza/2015 a & Zambia & November 2015 & MF622038 \\
\hline NDV/chicken/Mozambique/192A/2016 & Mozambique & March 2016 & KX231368 \\
\hline
\end{tabular}

${ }^{a}$ Isolated and sequenced in the present study 


\section{MATERIALS AND METHODS}

\section{Sampling and virus isolation}

Tissues were sampled at necropsy from chickens considered at risk of having ND by poultry veterinarians, based on suspicious clinical or autopsy findings. These birds included indigenous backyard chickens, broilers, broiler breeders and commercial layers. Samples were collected between August 2013 and November 2016 in South Africa, Zimbabwe, Malawi and Zambia. Viruses isolated in this study, and related sequences retrieved from the Genbank database are listed in Table 1. Isolations in specific pathogen free embryonated chicken eggs were performed by the Department of Veterinary Tropical Diseases at the University of Pretoria, Rainbow Veterinary Laboratory near Pietermaritzburg and Deltamune (Pty) Ltd. in Pretoria, according to the OIErecommended procedure (OIE, 2015). Total RNA was extracted from egg alantoic fluids with TRIzol ${ }^{\circledR}$ reagent (Life Technologies, Carlsbad, CA).

\section{Reverse transcription PCR and Sanger sequencing}

Oligonucleotide primer pair NDVF 4493for and NDVF5078rev was used to amplify the aminoterminal region of the genotype VIIh F protein-encoding gene; the 585-bp amplicon spanned the fusion protein cleavage site. Sequences are as follows: NDVF 4493for-

CACGGGTAGAAGAGTCTGGATCC; NDVF5078rev- GTGGCAGTTGGAAAGATGCAGCAG. For reverse transcription PCR, $3 \mu$ l of template RNA, 10pmol of each primer, $60 \mathrm{U}$ of Murine Leukemia Virus Reverse Transcriptase (Invitrogen, Carlsbad, CA, USA) and 12U of Ambion ${ }^{\circledR}$ RNAse Inhibitor (ThermoFisher Scientific, Waltham, MA, USA) was added to Phusion Flash PCR Master Mix (ThermoFisher Scientific). Reactions were incubated on a Verity 96 Well Thermal Cycler (Life Technologies, Carlsbad, CA, USA) in a 20 -minute reverse transcription step at $37^{\circ} \mathrm{C}$, followed by 35

cycles of a $53^{\circ} \mathrm{C}$ annealing temperature and 1 min elongation step. PCR products were separated by 
electrophoresis in $1 \%$ agarose stained with ethidium bromide. PCR amplicons were excised, purified with a QIAquick Gel Extraction kit (Qiagen, Hilden, Germany) and eluted products submitted to Inqaba Biotech (Pretoria) for Sanger sequencing, using the NDVF5078rev oligonucleotide. Genotype VIlh-specific nucleotide sequences were confirmed by BLAST homology searches (https://blast.ncbi.nlm.nih.gov/Blast.cgi).

\section{Library preparation and Illumina sequencing}

Transcriptomic libraries were generated as previously described (Abolnik et al 2012). Nextera libraries were prepared and analysed on an Illumina MiSeq apparatus by the sequencing service provider, Inqaba Biotech (Pretoria). Fusion protein sequences were extracted using an Assemble-ToReference approach in the CLC Genomics Workbench v. 5.1.5 (Qiagen, Aarhus, Denmark) and analysed further in BioEdit. Sequences were aligned and a Maximum Likelihood phylogenetic tree was constructed in MEGA v5.2 (Tamura et al 2011). Genome sequences were deposited in the GenBank database (Table 1).

Disease information for the specific countries was retrieved from the World Animal Health Information System (WAHIS), (OIE, 2017).

\section{Phylogenetic and Molecular clock analysis}

Phylogenetic trees of full gene sequences were constructed using the Maximum Likelihood inference in MEGAv5.2.2 software using the HKY model, Nearest Neighbour Interchange heuristic method, a very strong branch swap filter, and 1000 bootstrap replications to assign confidence levels to branches (Tamura et al., 2011). 
Time-stamped $\mathrm{F}$ protein gene sequences for which the months of collection were available (Table 1) were used to calculate dates to the most recent common ancestor. The BEAST program, version 2.3.2 (Drummond et al., 2012) uses a Bayesian Markov chain Monte Carlo (MCMC) approach. Various clock models and nucleotide substitution models were tested, but ultimately an HKY substitution model with estimated base frequencies, a Gamma site heterogeneity model with four gamma categories, uncorrelated lognormal relaxed clock and coalescent Bayesian skyline tree produced the best results. Uncertainty in the data is reflected in the $95 \%$ highest probability density (HPD) values for each parameter estimate, and the chain length was run for 80 million iterations to achieve coverage (effective sample size was $>400$ ) as assessed using the Tracer v1.5 program (Drummond et al., 2012). After $10 \%$ of the samples were removed as burn-in, the maximum clade credibility (MCC) phylogenetic tree was estimated from the posterior distribution of trees generated by BEAST using the program TreeAnnotator v2.3.2 (Drummond et al., 2012). The MCC tree was visualized using the program FigTree v1.3.1 (http://tree.bio.ed.ac.uk/software/figtree/), which allowed us to estimate the times of each individual node. 


\section{RESULTS}

\section{NCD in South Africa}

Up until the end of 2012 only genotype VIId strains were identified in NCD outbreaks in South Africa (Abolnik, 2017) and for the first six months of 2013, NCD was absent from most of the country except for the North-West and Limpopo provinces (Figs 1 and 2). NCD killed an entire flock of twelve backyard chickens in the North-West Province in February 2013, followed by further outbreaks of $100 \%$ mortalities in a flock of 7 backyard chickens in March and 10 backyard chickens in June. In June 2013, a single dead backyard chicken that had succumbed to NCD was reported in the Limpopo Province (OIE). None of these cases were submitted to the regional laboratory for F protein gene sequencing at the time.

Genotype VIIh in South Africa was initially confirmed by Sanger sequencing of a partial F protein gene region that was amplified using the oligonucleotide primer pair Alle/Alls described by Wang et al (2001). However, since this PCR amplifies most genotypes including vaccine strains and requires sequence confirmation of results, a genotype VIIh-specific PCR using the primer pair was developed for rapid diagnosis. Approximately fifty cases of genotype VIIh were confirmed in South Africa using this PCR method (results not shown), and the full F protein sequences were obtained on specific isolates selected for their geographic distribution and collection date (Table 1).

The first confirmed cases of genotype VIIh in South Africa were reported almost simultaneously. The index case on 23 August 2013 was detected in a flock of commercial layers displaying mild clinical signs. The location was Lephalale, a town in the Limpopo Province situated about 40km from the Botswana border (Fig. 1). Three days later, on the $26^{\text {th }}$ of August, a similar strain was detected in 31day old free range broilers near Potchefstroom, North West Province, some $400 \mathrm{~km}$ to the south, and these birds had marked proventricular haemorrhages. The third confirmed case, with significant mortalities, was in 13-day old broilers in Potchefstroom. Raised mortalities were also reported in 


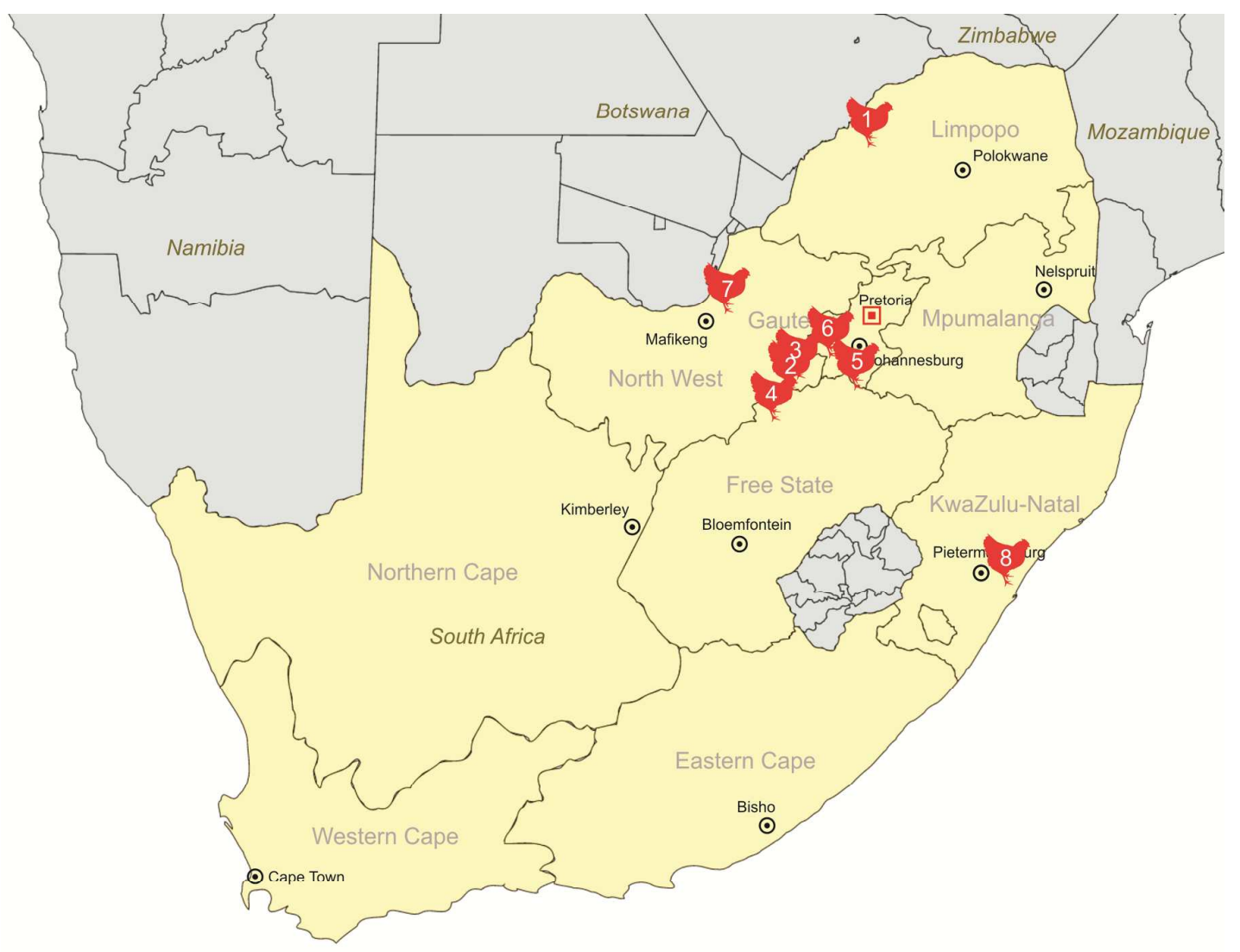

Figure 1. Locations of confirmed genotype VIIh Newcastle disease virus in outbreaks during the first six months of the epidemic in South Africa. 1-Lephalale (layers); 2 and 3-Potchefstroom (free-range broilers), 4- Viljoenskroon (broilers); 5-De Deur (backyard chickens); 6- Randfontein (layers); 7Zeerust (broilers); 8- Inchanga (backyard chickens). Subsequently, NDV spread to all provinces. 
broilers from Viljoenskroon sampled at the beginning of October 2013. On the $24^{\text {th }}$ of October, a single hen of a flock of four backyard chickens had died, and on necropsy proventricular, gastrointestinal and caecal tonsil haemorrhages were noted. The owner reported that a neighbour's turkey had been observed roosting with the hens, and suspected that the infection had been introduced to his birds in this manner. The isolate made from this hen, N2057, was originally reported as a "turkey" virus (Table 1). By the beginning of November 2013, genotype VIIh had been confirmed in a flock of commercial layers in Randfontein, and on the $20^{\text {th }}$ of November the virus was detected in a flock of 20 to 26-day old broilers near the town of Zeerust. The farm manager recorded depression in 30 to $50 \%$ of the birds, mild respiratory rales and an increase in mortalities from $25 \%$ up to $40 \%$. Severe proventricular and caecal tonsil haemorrhages were observed at necropsy. Around the same time a dead village chicken from Inchanga (in KwaZulu Natal Province) was submitted for laboratory diagnosis, and genotype VIIh was confirmed. In subsequent months, outbreaks persisted at other locations in these regions, but genotype VIIh subsequently spread throughout the country, presumably through the movements of cull chickens. By the end of 2014, all of South Africa's provinces had reported outbreaks, and these continued, with laboratory confirmation of the sole involvement of genotype VIIh to date (Fig. 2; OIE, 2017).

\section{NCD in Zimbabwe}

According to data reported to the OIE, an increased number of NCD outbreaks were reported in July 2013 in Zimbabwe's Manicaland Province (Figs. 2 and 3). Manicaland Province borders on Mozambique's Sofala Province where an outbreak in broiler chickens caused by genotype VIlh was also reported in July 2013 (Mapaco et al 2013). Outbreaks followed in Mashonaland Central and Midlands provinces in August. Although the types of poultry involved are not specified, susceptible flock sizes of between 500 and 800 birds suggest that smaller farms were involved. A report in Zimbabwe's Herald newspaper dated 7 October 2013 reported on outbreaks in the Domboshava region (Mashonaland East province) that had started in mid-September 2013 in indigenous, layer 


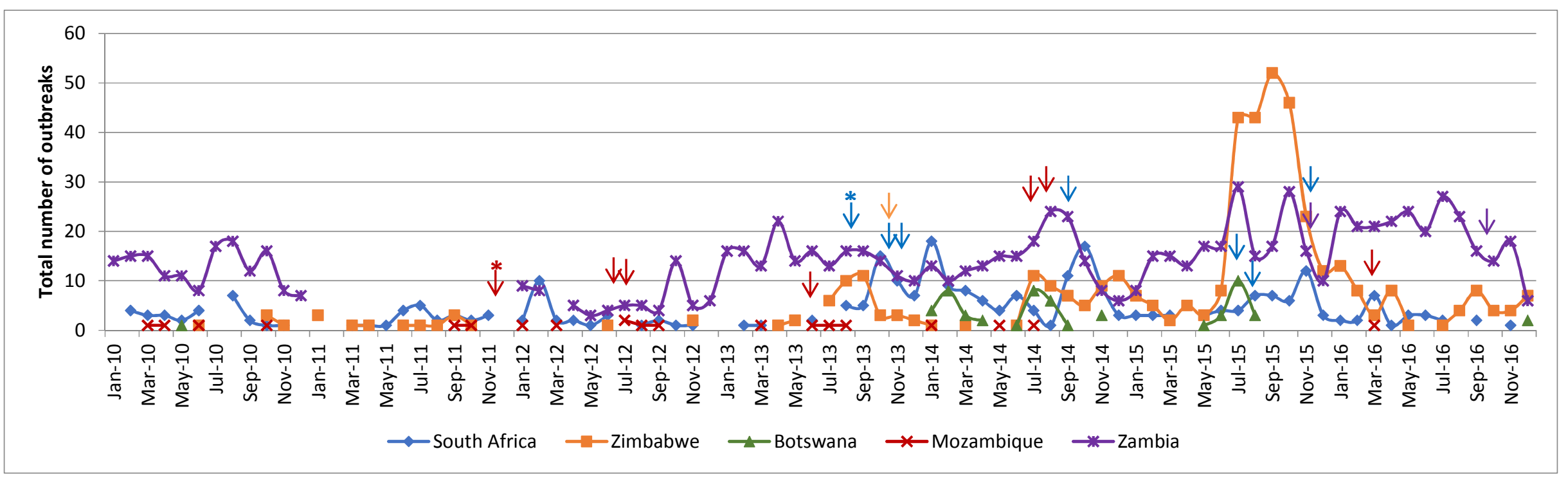

Figure 2. Newcastle disease outbreaks reported by southern African countries to the OIE from 2010-2016, according to the WAHID database. Arrows in the corresponding colour indicate cases of genotype VIIh confirmed by full F gene sequencing - see Table 1; *Earliest confirmed case. No data is available for Zambia for 2011. 
and broiler flocks in villages (The Poultry site, 2013). Villagers reported losing entire flocks of up to 100 chickens within days, and had resorted to the use of traditional medicines or slaughtering and eating the birds. In September 2013 the disease was also reported in Mashonaland West and Masvingo Provinces. Genotype VIlh strain 235280 was isolated from a commercial broiler flock in Harare in October 2013 (Table 1).

Outbreaks in Zimbabwe continued until 2016, with a substantial increase in cases in commercial operations from July to October 2015 (Figure 2). These outbreaks mainly occurred in the Masvingo Province, but Mashonaland Central was also severely affected by September 2015. The increased incidence of reported cases was mirrored in Botswana in July, Zambia in July and October, and South Africa in November 2015. July to October corresponds to late winter and early spring; the cool, dry windy season usually associated with NCD outbreaks and these climatic conditions are considered by farmers to help the virus spread.

\section{NCD in Zambia}

The incidence of NCD outbreaks reported in Zambia started to increase after September 2012 (Figure 2). The Copperbelt, Central, Luapula and Southern Provinces were mostly affected, but by early 2013 outbreaks were being reported from all provinces. In the Eastern Province of Zambia, 24 outbreaks were reported in 2015. As a result, sampling took place during active NCD outbreaks in the Chipata, Katete and Chadiza districts. Tracheal and cloacal swabs were collected from 17 dead and clinically sick indigenous chickens with paralysis, green diarrhoea, nasal and oral lacrimation, from which genotype VIlh was confirmed in November 2015. Genotype VIlh was also confirmed in commercial brown-egg layers near Ndola in October 2016. A genotype XIII strain was also however isolated from diseased indigenous backyard chickens in November 2015 (Abolnik et al., 2017), thus the outbreaks that occurred during this period cannot be attributed to solely genotype VIIh. 


\section{NCD in Malawi}

The Malawian veterinary authority does not make regular reports of disease incidence to the OIE, and therefore the data is not included in Fig. 2. In the first six months of 2013, five outbreaks were reported in an unspecified province. A single outbreak in Malawi was reported in the first six months of 2014 , but four outbreaks were reported in the second half of that year.

Two outbreaks caused by genotype VIIh in Malawi in September 2015 were confirmed by F protein gene sequencing from samples submitted to a private diagnostic company, MDS Africa (Durban, South Africa). This coincided with the increased outbreaks reported in Zimbabwe's commercial sector in the Mashonaland Central Province during the same period, located less than three hundred kilometres away (Figure 3). Both of these Malawi cases were in the Blantyre region of southern Malawi and involved commercial caged layers subjected to routine vaccination programmes including Newcastle disease. Drops in egg production, loss of egg colour and some thin-shelled or shell-less eggs were observed. Mortality was less than $5 \%$ with nervous signs and visceral lesions typical of NCD noted in some of the birds.

\section{Circumstantial evidence for genotype VIIh in Botswana}

An outbreak in backyard chickens in Serowe (Central Province) was reported to the OIE in early January 2014, followed by a cluster of outbreaks in Muchudi (Kgatleng Province), Gaborone (South East Province), Kanye (Southern Province) and Molepolole (Kweneng Province). Further outbreaks in Botswana's indigenous backyard flocks occurred throughout the winter months in 2014 and 2015. By comparing the timing of these outbreaks (Fig. 2) and their proximity to outbreaks in South Africa's Limpopo and North-West Provinces (Figs. 1 and 3) we consider it likely that Botswana's outbreaks were also caused by genotype VIIh, either spreading from Botswana to South Africa or vice versa through the movement of infected backyard poultry or spent hens. Unfortunately, Botswana's 
National Veterinary Laboratory declined to participate in the present study, but we anticipate that sequencing of archived samples will confirm these cases as genotype VIlh at a later stage.

\section{Phylogeny and dating of recent common ancestors}

Full F protein sequences for all available global genotype VIIh strains were phylogenetically analysed, with representative sequences from southern Africa listed in Table 1. Vietnamese genotype VIIh strains were not included in the phylogenetic or BEAST analysis because only partial sequences of $<300$ bp were available (Choi et al., 2014).

Chinese viruses isolated in 2012 were basal to the southern African strains (Figure 4). Within the southern African cluster, two separate clusters separated by a bootstrap value of 67 are evident. The first is a cluster containing only South African viruses isolated from 2013 to 2015 , and a single isolate from Mozambique, strain 192A, collected during an outbreak in 2016 that affected a flock of 350 broilers with a $71.4 \%$ mortality rate (Mapaco et al., 2016). Mozambique strain 192A is very closely related to South African strain 32995, isolated in July 2015 (99.9\% nucleotide sequence similarity), suggesting that the infection spread from South Africa across the eastern border to Maputo in this instance. South Africa exports point-of-lay hens and day-old chicks to Mozambique, and cull chickens (spent hens and live broilers) are also likely to cross the border.

The second southern African cluster contains the majority of the viruses from Mozambique and the Zimbabwean strain 235280 that was isolated in 2013. This cluster shares an RCA with strain 662 from Mozambique, isolated in the Zambesia province in August 2014 (Mapaco et al 2016). Mozambique's Zambezia province borders on Malawi (Fig. 3). The three Zambian viruses isolated at Chadiza, Katete and Chipata (Mbeweka) in November 2015 have comparatively long branch lengths that are associated with extensive mutations over time. All three Zambian strains however share a root, and thus a common ancestor/s. In addition, the Zambian strains share an RCA with Mozambique strain 


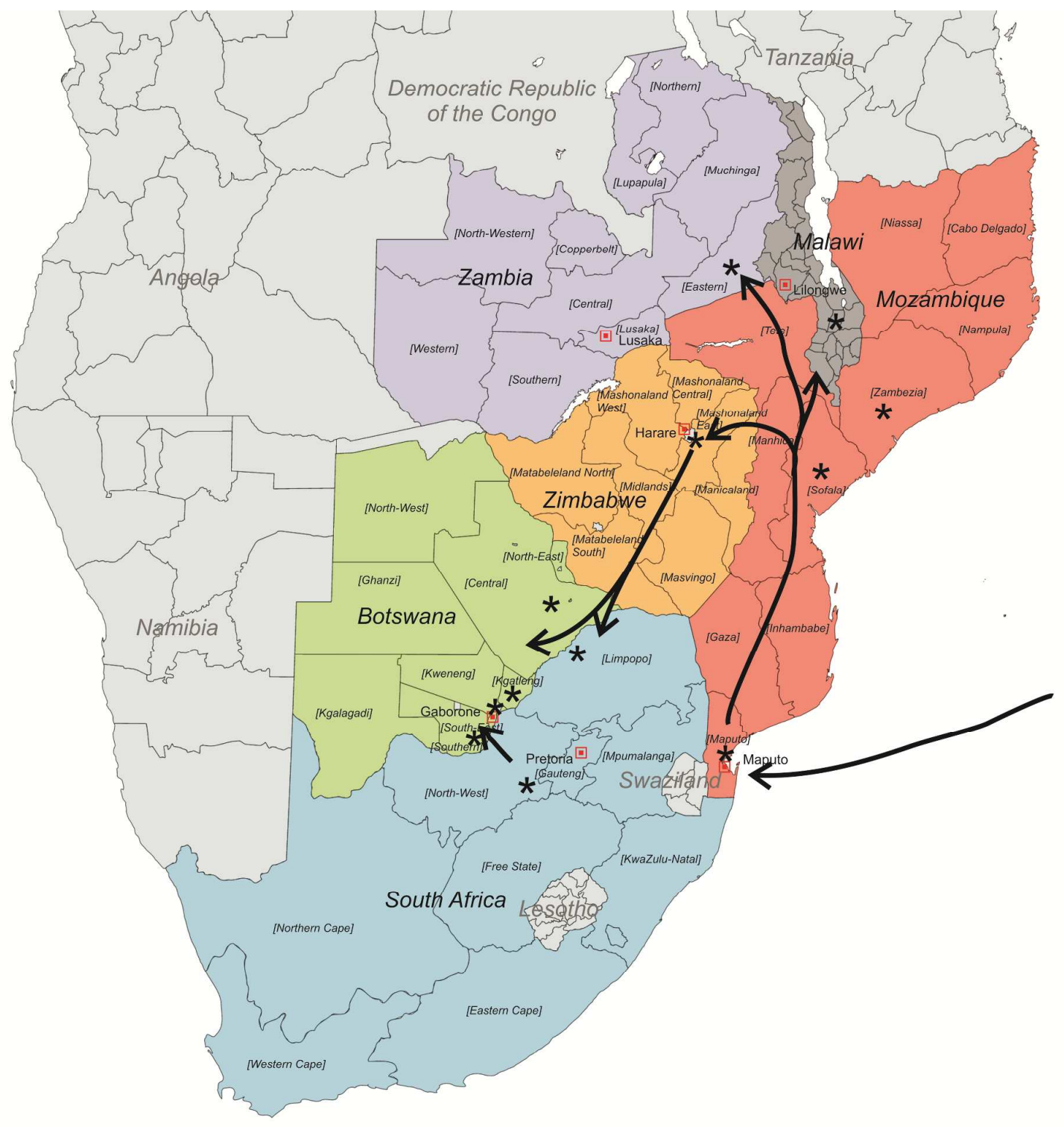

Figure 3. Southern African provinces affected by genotype VIlh Newcastle disease outnreaks, 20132016. The locations of confirmed outbreaks either reported to the OIE or identified in the present study are indicated by *. The arrows indicate suspected routes of spread in the sub-region based on timing of disease reports and molecular dating. 
622 from the Zambezia province. This topology suggests that Mozambique strains were the sources of outbreaks in both Zimbabwe and Zambia. The F protein sequences for Malawian strains were not available for comparison.

To establish when Newcastle viruses were introduced into each of the countries, we estimated the time to the most recent common ancestor (tMRCA). The time-scaled tree showing the 95\% HPDs on the branches is provided in Fig 5. The tMRCA for Chinese and all African viruses was dated at August 2010 (July 2008-April 2011), the tMRCA for the clade of all African viruses (Mozambique, Zambia, Zimbabwe and South Africa) is dated at January 2011 (Sept 2009-Oct 2011), and the tMRCA for the clade that contains the Mozambique viruses, along with Zambian and Zimbabwean strains is dated at January 2011 (June 2010-November 2011). This suggests that the RCA of the genotype VIlh viruses that were initially transferred to Africa from south East Asia arose sometime between August 2010 and January 2011, and that it was possibly introduced to Maputo province between January and June 2011. The earliest confirmed case of genotype VIIh in Mozambique was recovered in Maputo province on 12 December 2011 (Mapaco et al 2016), but in September and October 2011, the Mozambique veterinary authority reported two large outbreaks in domestic chickens, in flocks of approximately 27000 birds, with mortality rates varying between 11.6 and 22.9\% (Fig 2, OIE WAHIS). The dating points to the much earlier introduction of genotype VIIh into Mozambique, within the first six months of 2011. Mapaco et al (2016) suggested illegal poultry trade as the source of the outbreak as no day-old breeder chicks are imported from Asia. Since no outbreaks were recorded in commercial flocks prior to September 2011 and the RCA is dated to much earlier in 2011, Maputo Province's backyard flocks were the likely entry point for genotype VIIh in Africa.

Next, we examined the spread of the virus within Africa, based on estimation of the RCA dates. The tMRCA for Mozambique strain 658 and all Zambian and Zimbabwe isolates, as well as strains 475 and 622 from Mozambique, is April 2012 (July 2011-Aug 2012). The tMRCA for Mozambique strain 622 and Zimbabwean strain 235280 is dated at September 2013 (Jan 2012-Sept 2013), and the 
tMRCA for Mozambique strain 475 and the Zambian strains from Katete, Mbeweka and Chidiza is dated at October 2013 (Sept 2012-July 2014). After the initial detection and identification of genotype VIIh in Mozambique in late 2012, the virus reached Mozambique's Zambezia province further up the East African coast by August 2014. However, dating suggests that the progenitors to the strains isolated in Zambia and Zimbabwe were already present outside of the Maputo province around September/ October 2013. The tMRCA for the three Zambian strains is dated at March 2014 (Jan 2013-Aug 2015). It was in March 2014 that the frequency of NCD outbreaks in Zambia started to increase, according to what was reported to the OIE, with peaks around August 2014.

The tMRCA for the South African clade is dated at December 2012 (Nov 2011-July 2013). The infection was not present in commercial operations at the time, but reports of disease in the backyard flocks of the North-West Province, starting in February 2013 are consistent with this dating. Two sub-clades visible in both Fig 4 and Fig 5, with tMRCAs dated at March 2013 (June 2012October 2013) and June 2013 (Dec 2012-July 2013) lack statistical support, thus the possibility of two separate introductions into South Africa around April and June 2013 cannot be ruled out.

Nonetheless, the phylogenetic evidence (i.e. Mozambique strains with higher sequence diversity, group more closely with strains from Zimbabwe and Zambia) does not support an early direct transmission event from Mozambique to South Africa, but rather implies that backyard flocks from either Zimbabwe or Botswana were the source. 


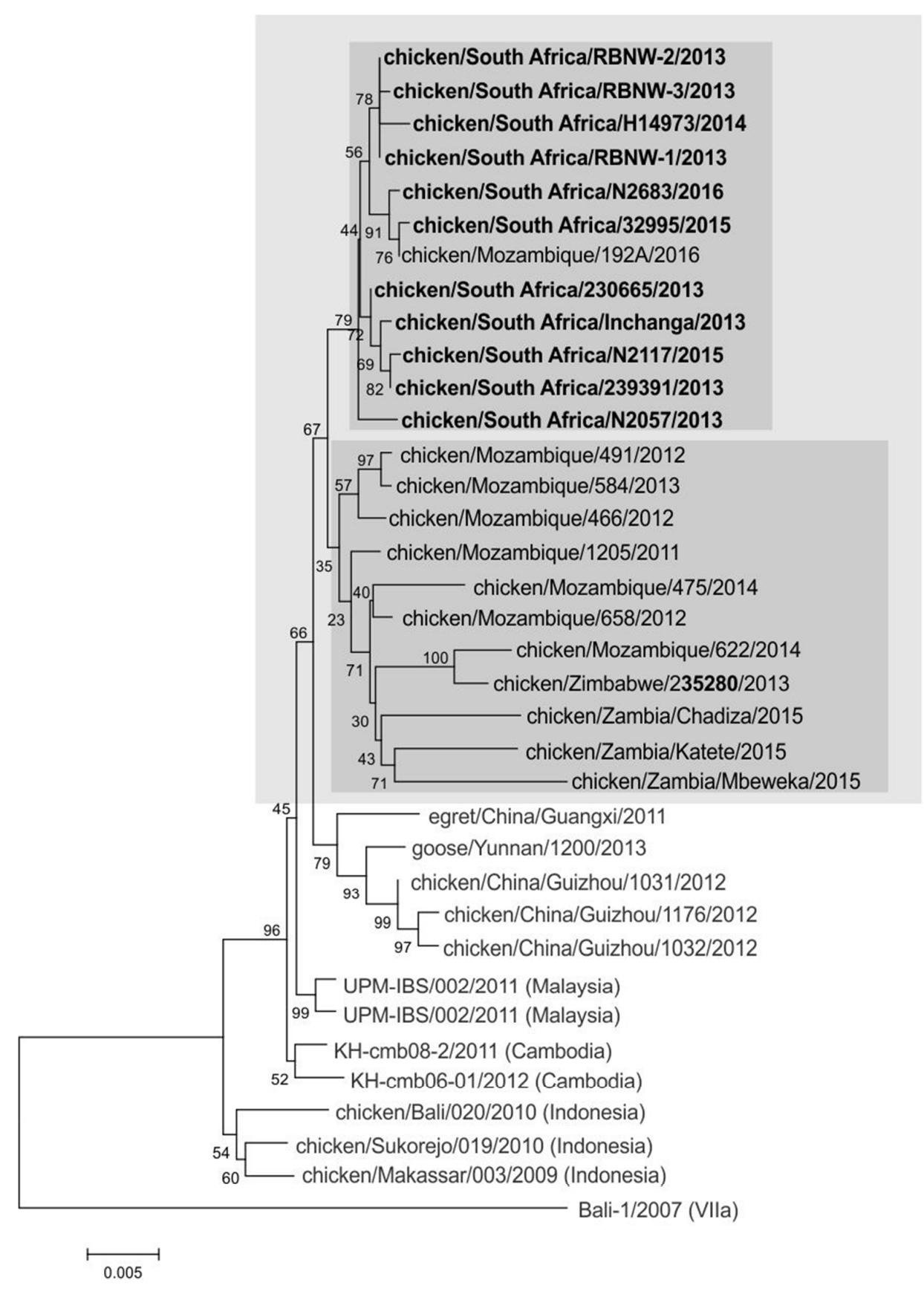

Figure 4. Maximum likelihood tree of fusion protein gene sequences. 


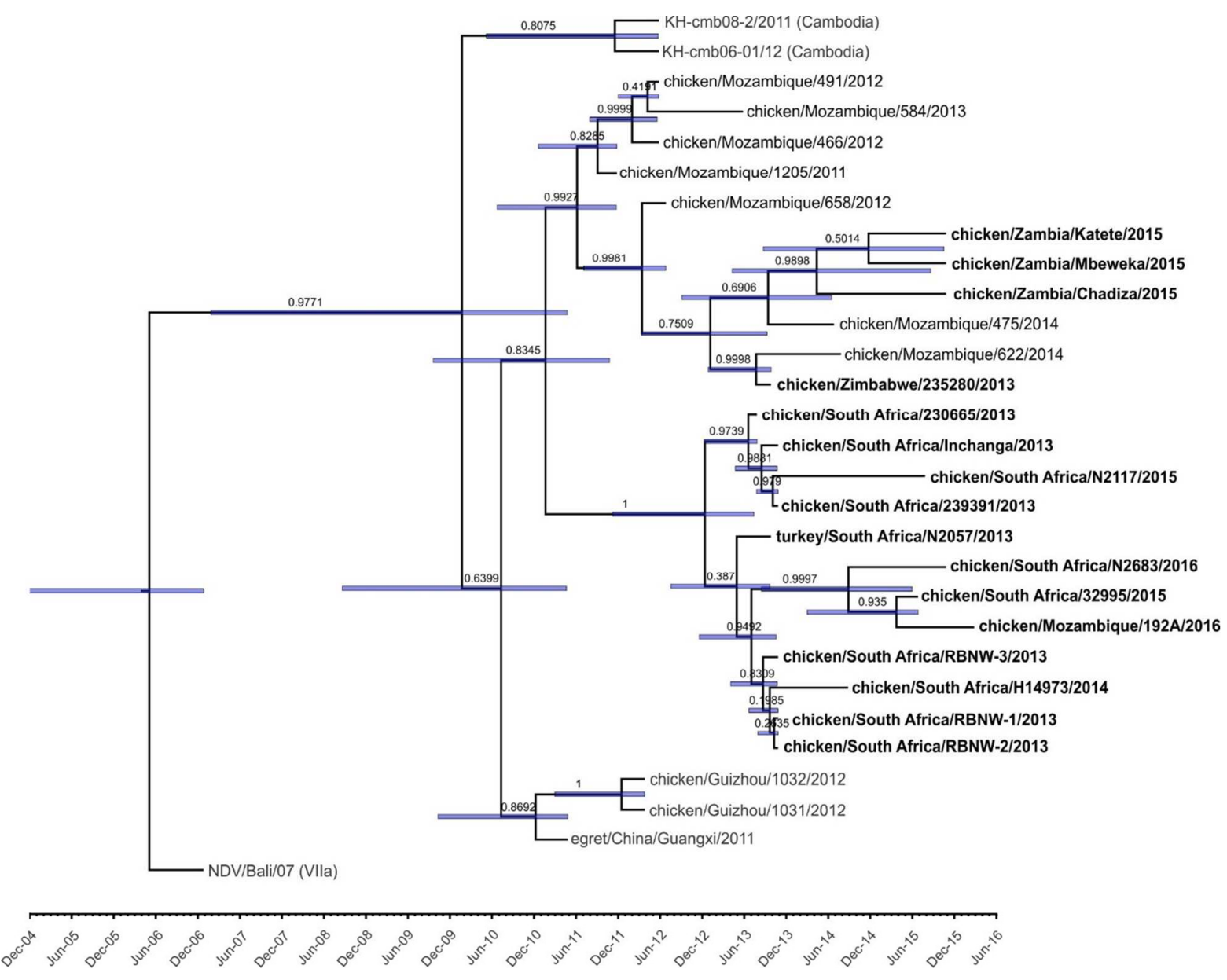

Figure 5. Time-scaled phylogeny of the Newcastle disease virus fusion protein genes, with posterior probabilities indicated in blue on the branches. Sequences generated in this study are highlighted. 


\section{DISCUSSION}

Southeast Asia represents one of the largest concentrations of commercial and backyard poultry in the world. Mixed poultry production systems with a large portion of the birds raised in backyard farms, and an abundance of live bird markets selling birds of multiple species and ages favours the emergence of genetically diverse virulent NDV isolates, i.e. new sub-genotypes (Miller et al 2015). We used a combination of phylogenetic evidence, molecular dating and epidemiological reports to trace the origins of sub-genotype VIIh Newcastle disease from Southeast Asia to and within southern Africa. The collated results were used to illustrate the likely path of the infection in Fig. 3: after the viruswas introduced into the backyard flocks around the port of Maputo in Mozambique in early 2011, it infected commercial flocks in Mozambique (Mapaco et al), spreading northwards through Mozambique, and then into the backyard poultry of the north eastern provinces of Zimbabwe. From Mozambique, it reached neighbouring Malawi and Zambia. In Zimbabwe, the disease spread southward towards South Africa and Botswana, causing outbreaks in backyard chickens in early to mid-2013. Exchange of poultry across the South Africa-Botswana border was a likely source of the infection. In August 2013 the disease entered South Africa's large commercial industry, and the entire country was infected within a year, likely through fomites and the movements of cull chickens. At least one case of genotype VIIh being spread from South Africa back to Mozambique in 2015 was detected.

The Port of Maputo is strategically located between industrial areas of Southern Africa and major economic and industrialized regions of Eastern and Southern Asia, with direct road and rail connections to South Africa, Swaziland and Zimbabwe. Mozambique does not officially import dayold chicks from Asia, thus illegal trade was considered to be the likely source of genotype VIIh (Mapaco et al 2016). Illegal swill dumping that can be scavenged by humans and backyard poultry is another possibility. Mapaco et al (2016) suggested that migratory wild birds should also be considered as a possible source of the infection, through cross-overs occurring between the East 


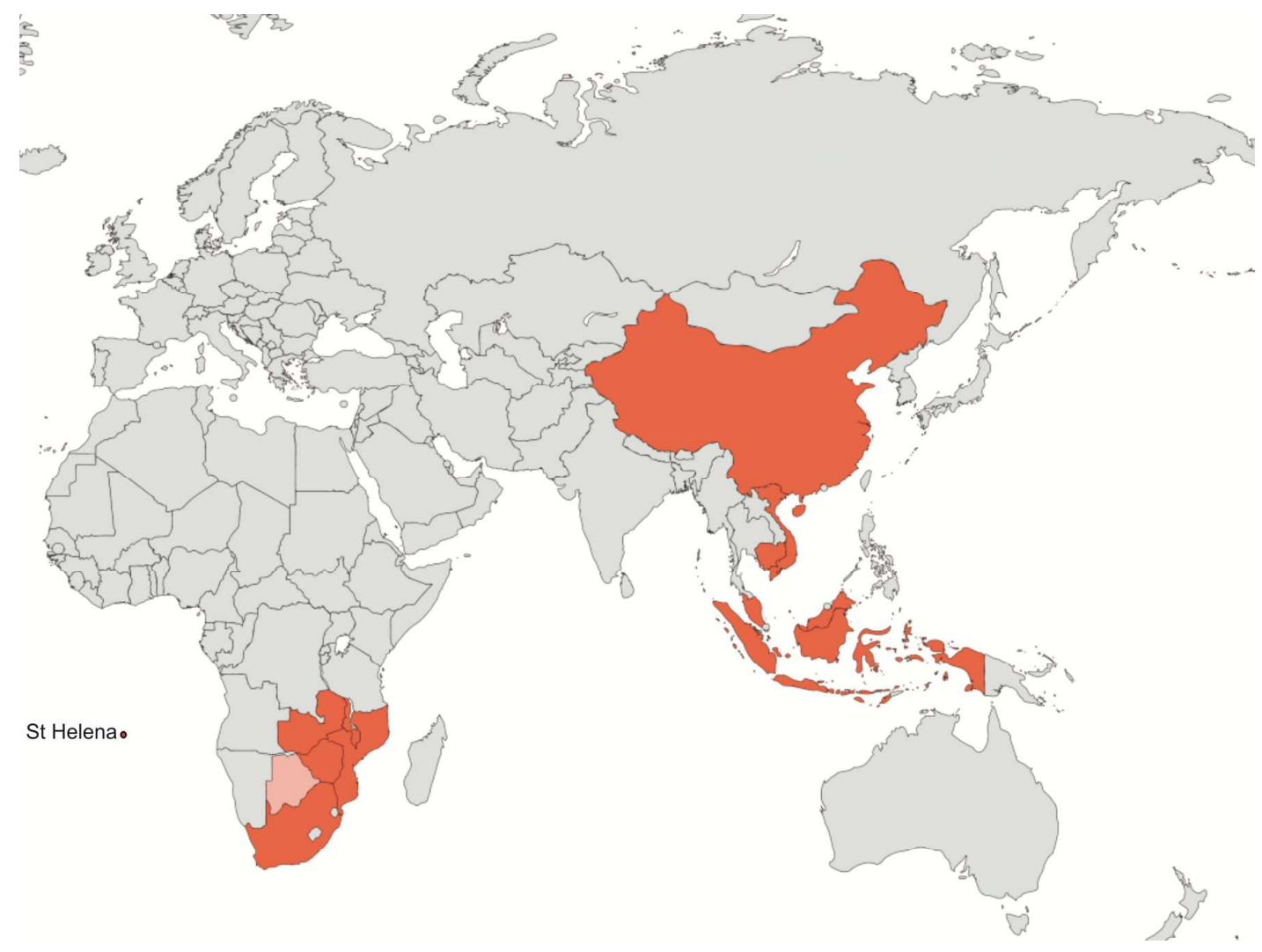

Figure 6. Global distribution of Newcastle disease genotype VIIh virus, 2011-2016. Presence in Botswana (lighter shade) is suspected but unconfirmed. 
Africa, West Asian and East Australian flyways. Forty four Palearctic migrant species that overwinter in southern Africa migrate from their Siberian breeding grounds through Russia, Eastern Europe and the Middle East down through East Africa's Rift Valley.

(http://www.birdlife.org.za/conservation/migratory-birds). In Fig 6, the current global distribution of genotype VIlh is illustrated, with no reports of the virus in the poultry of Russia, Eastern Europe, or the Middle East. The lack of genotype VIlh outside of Southeast Asia and southern Africa is the strongest indicator that wild migratory birds were not the carriers. Instead, the movement by ship of infected poultry or fomites from Southeast Asia to Maputo's port is the most likely scenario. The dissemination of exotic Newcastle disease by ship is lent credence by reports of NCD outbreaks in backyard chickens on the island of St Helena. St Helena is situated approximately $2000 \mathrm{~km}$ off the south western African coast, and lies on a shipping route around the southern tip of the African continent (Fig. 6). The cause of the outbreaks in backyard chickens on St Helena between August 2014 and November 2014 (OIE, 2017), was identified as genotype VIIh at AHPA, VLA (Ruth Manvell, personal communication). The genetic sequence isn't yet publically available for comparison, but it would be interesting to see whether the strain was closer in genetic sequence to those isolated from South Africa, or Mozambique around the same time.

This study demonstrates how vulnerable African countries remain to exotic sources of infection, and how quickly disease spreads within a region. It is widely accepted that ND is endemic in most African countries, but little attention has been given to establishing the sources and frequency of the introductions of exotic strains. These introductions have a high cost in Africa. Whereas exotic Newcastle disease outbreaks cause serious financial losses to small, medium and large commercial enterprises, ND outbreaks have devastating consequences for the poorest households. Poultry are often raised by women and children, the most vulnerable members of the community. In addition to providing high-quality protein in the household, income from the sale of poultry is used as a ready means of cash to buy books and other school requirements as poultry can be sold more quickly than other livestock (Songolo and Katongo, 2000). 
The commercial poultry sector and smaller farming operations in Africa have enormous potential to stimulate socio-economic growth. Likewise, backyard chickens fulfil very important roles in the daily lives of many households. Identifying the sources of outbreaks is a first step in tightening controls, but other interventions are required to prevent and control exotic ND from being introduced and spreading in Africa. Stricter biosecurity controls at borders and ports, more accessible vaccination for small scale farmers and backyard poultry, and raising the public's awareness about how ND is spread and how to safeguard and protect their flocks is required. The identification of important trade hubs where surveillance can be focussed and better engagement of farmers in reporting disease outbreaks has been shown to be an effective way of more rapidly detecting outbreaks in Eastern Zambia and thus preventing spread of disease (Mubamba et al, 2017). Application of similar strategies in other SADC countries may go a long way to preventing such widespread propagation of Newcastle disease in future. In commercial flocks, more attention is needed to ensure that flocks respond uniformly and with a good immune response to applied vaccination programmes.

\section{ACKNOWLEDGEMENTS}

The authors thank Adrian Knoetze, I-Chang Lee, Dionne Rauff, Christine Strydom, Telana Rapp, Karen Ebersohn, Thandeka Phiri and Peter Smith for samples or excellent technical assistance. The study was funded by South African National Research Foundation grant \#CPRR 93461.

\section{CONFLICT OF INTEREST STATEMENT}

The authors declare no conflict of interest 


\section{RERERENCES}

Abolnik, C. (2017). History of Newcastle disease in South Africa. Onderstepoort Journal of Veterinary Research 84(1), e1-e7

Abolnik, C., Olivier, A.J., Grewar, J., Gers, S., \& Romito, M. (2012). Molecular analysis of the 2011 HPAI H5N2 outbreak in ostriches, South Africa. Avian Dis. 56(4 Suppl), 865-879.

Abolnik, C., Mubamba, C., Dautu, G., \& Gummow, B. (2017). Complete genome sequence of a Newcastle disease genotype XIII virus isolated from indigenous chickens in Zambia. Genome Announcements. In press.

Adi, A.A., Astawa, N.M., Putra, K.S., Hayashi, Y. \& Matsumoto, Y. (2010). Isolation and characterization of a pathogenic Newcastle disease virus from a natural case in Indonesia. J Vet Med Sci. 72(3), 313-319.

Alexander, D.J. (2000). Newcastle disease and other avian paramyxoviruses. Rev Sci Tech 19, $443-$ 462.

Choi, K-S., Kye, S-J., Kim, J-Y, Damasco, V., Sorn, S., Lee, Y-J., Choi, J-C., Kang, H-M., Kim, K-I., Song, BM. \& Lee, H-S. (2013). Molecular epidemiological investigation of velogenic Newcastle disease viruses from village chickens in Cambodia. Virus Genes 47, 244-249.

Choi, K-S., Kye, S-J., Kim, J-Y, To, T.L., Nguyen, D.T., Lee, Y-J., Choi, J-C., Kang, H-M., Kim, K-I., Song, BM. \& Lee, H-S. (2014). Molecular epidemiology of Newcastle disease viruses in Vietnam. Trop Anim. Health Prod. 46, 271-277.

Diel, D.G., da Silva, L.H., Liu, H., Wang, Z., Miller, P.J. \& Afonso, C.L. (2012), Genetic diversity of avian paramyxovirus type 1: proposal for a unified nomenclature and classification system of Newcastle disease virus genotypes.Infect Genet Evol. 12(8), 1770-1779. doi: 10.1016/j.meegid.2012.07.012. 
Drummond, A.J., Suchard, M.A., Xie, D. \& Rambaut, A. (2012) Bayesian phylogenetics with BEAUti and the BEAST 1.7 Molecular Biology And Evolution 29, 1969-1973

Hall, T.A. (1999). BioEdit: a user-friendly biological sequence alignment editor and analysis program for Windows 95/98/NT. Nucl. Acids. Symp. Ser. 41, 95-98.

Mapaco, L.P., Monjane, I.V., Nhamusso, A.E., Viljoen, G.J., Dundon, W.G. \& Achá, S.J. (2016).

Phylogenetic analysis of Newcastle disease viruses isolated from commercial poultry in Mozambique (2011-2016). Virus Genes 52(5), 748-753.

Miller, P.J., Haddas, R., Simanov, L., Lublin, A., Rehmani, S.F., Wajid, A., Bibi, T., Khan, T.A., Yaqub, T., Setiyaningsih, S. \& Afonso, C.L. (2015). Identification of new sub-genotypes of virulent Newcastle disease virus with potential panzootic features. Infect Genet Evol. 29, 216-229.

Molini, U., Aikukutu, G., Khaiseb, S., Cattoli, G. \& Dundon, W.G. (2017). First genetic characterization of Newcastle disease viruses from Namibia: identification of novel VIIk subgenotype. Arch Virol 162(8), 2427-2431. doi: 10.1007/s00705-017-3389-y

Mubamba C., Ramsay G., Abolnik C., Dautu, G. \& Gummow, B. (2017). Using syndromic data from rural poultry farmers as a viable poultry disease reporting tool and means of evaluating remedies for poultry diseases in Eastern Zambia. Submitted.

OIE Manual of Diagnostic Tests and Vaccines for Terrestrial Animals (2015). Chapter 2.3.1.4.

Newcastle disease (Infection with Newcastle disease virus). Accessed at:

http://www.oie.int/fileadmin/Home/eng/Health_standards/tahm/2.03.14_NEWCASTLE_DIS.pdf

OIE World Animal Health Information Database (WAHID) Interface (2017). Accessed at:

https://www.oie.int/wahis 2/public/wahid.php/Diseaseinformation/diseasehome.em/data-after2004-wahis-interface/ 
The Poultry Site (2013). Accessed at:

http://www.thepoultrysite.com/poultrynews/30257/suspected-newcastle-disease-outbreak-inzimbabwean-province/

Songolo, A. \& Katongo, J.C. (2000). 'Country report: Zambia'. In: Alders, R.G., Spradbrow, P.B. (Eds.), SADC planning workshop on Newcastle disease control in village chickens: Proceedings of an international workshop, ACIAR Proceedings 103, Maputo, Mozambique. 43-45

Tamura, K., Peterson, D., Peterson, N., Stecher, G., Nei, M. \& Kumar, S. (2011). MEGA5: Molecular Evolutionary Genetics Analysis Using Maximum Likelihood, Evolutionary Distance, and Maximum Parsimony Method. Molecular Biology and Evolution 28, 2731-2739.

Tan, S.W., Omar, A.R., Aini, I., Yusoff, K. \& Tan, W.S. (2004). Detection of Newcastle disease virus using a SYBR Green I real time polymerase chain reaction. Acta Virol. 48(1), 23-28.

Wang, Z., Vreede, F.T., Mitchell, J.O. \& Viljoen, G.J. (2001). Rapid detection and differentiation of Newcastle disease virus isolates by a triple one-step RT-PCR. Onderstepoort J Vet Res. 68(2), 131134.

Xiao, S., Paldurai, A., Nayak, B., Samuel, A., Bharoto, E.E., Prajitno, T.Y., Collins, P.L. \& Samal, S.K. (2012). Complete genome sequences of Newcastle disease virus strains circulating in chicken populations of Indonesia. J Virol. 86(10), 5969-5970.

Xie, Z., Xie, L., Chen, A., Liu, J., Pang, Y., Deng, X., Xie, Z. \& Fan, Q.(2012). Complete genome sequence analysis of a Newcastle disease virus isolated from a wild egret. J Virol. 86(24):13854-13855. doi:

10.1128/JVI.02669-12. 